\title{
Efecto de la aplicación de tres dosis de Bacillus subtilis en tres variedades de fréjol arbustivo
}

\section{Effect of applying three dosages of Bacillus subtilis on three bush beans varieties}

Melanie Arline Chávez Rea1. José Eliécer Vásquez-Guzmán ${ }^{2 *}$

\footnotetext{
${ }^{1}$ Universidad Central del Ecuador, Facultad de Ciencias Agrícolas, Carrera Ingeniería Agronómica Jerónimo Leiton y Av. La Gasca s/n, Ciudadela Universitaria. Quito. 170521. Ecuador.

$\otimes$ machavezr@uce.edu.ec

Q. https://orcid.org/0000-0002-4107-8977

${ }^{2}$ Universidad Central del Ecuador, Facultad de Ciencias Agrícolas, Carrera Ingeniería Agronómica Jerónimo Leiton y Av. La Gasca s/n, Ciudadela Universitaria. Quito. 170521. Ecuador.

$\bowtie$ jevasquezg@uce.edu.ec

Q https://orcid.org/0000-0001-6290-1419
}

*Autor de correspondencia: jevasquezg@uce.edu.ec

\section{SIEMBRA}

https://revistadigital.uce.edu.ec/index.php/SIEMBRA ISSN-e: $2477-8850$

ISSN: $1390-8928$

Periodicidad: semestral

vol. 8, núm. 2, 2021

siembra.fag@uce.edu.ec

DOI: https://doi.org/10.29166/siembra.v8i2.2657

Esta obra está bajo una licencia internacional Creative Commons Atribución-NoComercial

\section{Resumen}

El objetivo de la presente investigación fue evaluar el efecto de la aplicación de tres dosis de Bacillus subtilis en tres variedades de fréjol arbustivo (Phaseolus vulgaris L.), para determinar la respuesta en rendimiento y otras variables. Se realizó la aplicación de tres dosis: D1 sin aplicación (testigo), D2 $250 \mathrm{ml}$ y D3 $500 \mathrm{ml}$, del inoculante biológico NITO (Bacillus subtilis) disueltos en 200 L de agua, en las variedades de fréjol Paragachi, Campeón y Centenario. El ensayo se implementó en el Campo Académico Docente Experimental La Tola, mediante arreglo de parcelas divididas dispuestas en diseño de bloques completos al azar con tres repeticiones. Se evaluaron las variables agronómicas, días a la cosecha, vainas planta ${ }^{-1}$, granos vaina ${ }^{-1}$, peso de 100 granos y rendimiento en $\mathrm{kg} \mathrm{ha}^{-1}$. El mejor resultado obtuvo la interacción de la dosificación de $500 \mathrm{ml}$ y la variedad Centenario con un rendimiento de $3.763,89 \mathrm{~kg} \mathrm{ha}^{-1}$, la misma que refleja ser económicamente rentable con una ganancia neta de 0,32 (32 ctvs.) por dólar invertido. De la misma forma, se observaron diferencias en la concentración de fósforo disponible por acción de Bacillus subtilis, siendo la dosis D3 la que presentó mayor concentración de fósforo con 351,97 ppm.

Palabras clave: rendimiento, fósforo, fitopatógenos, radiculares.

\begin{abstract}
The objective of this research was to evaluate the effect of applying three dosages of Bacillus subtilis to three varieties of beans (Phaseolus vulgaris L.) to determine an increase in yield and in other traits. Three dosages were applied, D1 without apliction (check), D2 $250 \mathrm{ml}$ and D3 $500 \mathrm{ml}$, of biological inoculant NITO (Bacillus subtilis), dissolved in $200 \mathrm{~L}$ of water to Paragachi, Campeón and Centenario varieties. The trial was sat up in the Campo Académico Docente Experimental La Tola, using an split plot arrangement in a experimental Randomized complete block design, with three replications. The following traits were evaluated: days to harvest, pods per plant-1, beans per pod-1, weight of 100 seeds, and yield in $\mathrm{kg}$ per ha- 1 . The highest yield got the interaction $500 \mathrm{ml}$ dose $\mathrm{x}$ Centenario variety with a seed yield of $3763.89 \mathrm{~kg}$ ha-1 providing an economic profitability of 1.32 dollars. Likewise, differences were observed in concentration of available phosphorus due to the Bacillus subtilis, with dosage 3 providing the highest concentration of phosphorus with $351.97 \mathrm{ppm}$.
\end{abstract}

Keywords: seed yield, phosphorus, plant pathogens, roots 


\section{Introducción}

El fréjol (Phaseolus vulgaris L.), es una planta leguminosa del orden Fabales y familia Fabaceae. Es un alimento de uso común en varios países del mundo, por su accesibilidad al no ser costoso y por poseer varios nutrientes en los que destacan las proteínas, carbohidratos y hierro que puede sustituir al consumo de carne (Garcés Fiallos et $a l ., 2013)$. Por otra parte, el fréjol representa ingresos económicos para pequeños y medianos productores, ocupando el octavo lugar entre las leguminosas más sembradas en el mundo ( Garcés Fiallos et al., 2012).

Según Akibode y Maredia (2011) los mayores productores de esta leguminosa se encuentran en la India (3,40 millones ton), Brasil (3,36 millones ton), Myanmar (2,50 millones ton), China (1,60 millones ton) y México (1,17 millones ton). En todo el mundo se producen aproximadamente 21 millones de toneladas de este alimento. Ecuador produce 26.498 ton, es decir, el 0,2 \% de la producción mundial. En el Ecuador, de esta leguminosa se cosecha 7.018 ha en grano fresco y 24.006 ha en grano seco. De la misma forma se cosecha 16.367 ha en monocultivo y de forma asociada se cosecha 14.657 ha (Instituto Nacional de Estadística y Censos [INEC], 2019). Las pérdidas de cosechas se presentan por la escasa disponibilidad de variedades mejoradas, semillas de mala calidad, incidencia de plagas y enfermedades, y manejo inadecuado del cultivo.

Por otro lado, el rendimiento promedio de fréjol registrado en Ecuador es bajo con $430 \mathrm{~kg} \mathrm{ha}^{-1}$ en monocultivo y $110 \mathrm{~kg} \mathrm{ha}^{-1}$ en asociación con maíz, frente al rendimiento potencial del cultivo que sobrepasa los $2.000 \mathrm{~kg} \mathrm{ha}^{-1}$ (Torres Navarrete et al., 2013). En la agricultura se ha buscado obtener rendimientos altos por unidad de superficie, con el fin de satisfacer las necesidades alimenticias de la población. Lo anterior ha llevado al uso de fertilizantes químicos y pesticidas para aumentar la productividad. Sin embargo, esto no ha sido totalmente logrado ya que hasta la fecha los rendimientos en Ecuador siguen siendo bajos como lo señala Armenta-Bojórquez et al. (2010).

Corrales Ramírez et al. (2017), afirman que uno de los desafíos de la agricultura es hacer un uso más eficiente de los recursos para hacer frente al aumento de la demanda de alimentos. Como resultado de una producción agrícola intensiva y fertilización desbalanceada se ha reportado baja disponibilidad de potasio debido a una disminución en las reservas en el suelo y baja solubilización de fertilizantes fosfatados en la tierra.

Los microorganismos que se encuentran en los biofertilizantes realizan varios de los ciclos biológicos y químicos existentes, por lo que las funciones que efectúan son fundamentales para mantener el equilibrio en los agroecosistemas. Estas poblaciones microbianas cumplen una actividad importante, ya que en muchos suelos se encuentran grandes reservas de fósforo en formas no disponibles (Llanos, 2017). Diferentes estudios, en el sector agrícola, han demostrado que el uso de microorganismos en biofertilizantes promueve la erradicación de los fertilizantes químicos y el incremento de los rendimientos, representando un impacto positivo sobre el ambiente (Terry et al., 2005).

Según Corrales Ramírez et al. (2017) y Vidal (2003), Bacillus subtilis ha sido estudiado por poseer una variedad de mecanismos, entre ellos la solubilización de fosfatos y enzimas que contribuyen a la disponibilidad del elemento, producción de metabolitos secundarios como auxinas (ácido indolacético), producción de antibióticos, lipopéptidos, sideróforos y activación de la señalización de la respuesta sistémica inducida por producción de compuestos volátiles como ácido jasmónico y etileno, que ayudan a la actividad antagónica e inhibidora de hongos fitopatógenos del suelo.

Considerando que el rendimiento de fréjol arbustivo en el Ecuador es bajo, por el inadecuado manejo del cultivo, se propone el uso de Bacillus subtilis como alternativa. Con este microorganismo se pretende mantener el equilibrio microbiano del suelo y promover el desarrollo mediante la solubilización del fósforo.

Por tanto, el objetivo general de la investigación fue evaluar el efecto de la aplicación de tres dosis de Bacillus subtilis en tres variedades de fréjol arbustivo. Los objetivos específicos fueron establecer la variedad de fréjol que genere mayor rendimiento en respuesta a la aplicación del producto. Además, esta sería una alternativa económica, ecológica y productiva.

\section{Materiales y métodos}

\subsection{Localización}

La investigación se realizó en el Lote 2.1., perteneciente al "Área de horticultura", en el Campo Académico Docente Experimental La Tola, ubicado en la parroquia Tumbaco del cantón Quito. Sus coordenadas geográficas son $00^{\circ} 13^{\prime} 49^{\prime \prime}$ latitud Sur y $78^{\circ} 21^{\prime} 18^{\prime \prime}$ longitud Oeste, a una altitud de $2.460 \mathrm{~m}$. La temperatura promedio anual es de $16^{\circ} \mathrm{C}$ y la precipitación promedio anual es de $927 \mathrm{~mm}$. El área neta del experimento fue de $459 \mathrm{~m}^{2}$ con diseño experimental de Bloques completos al azar con arreglo en parcela dividida. 


\subsection{Materiales utilizados}

Se utilizó semilla de tres variedades de fréjol arbustivo: Paragachi, Campeón y Centenario. Paragachi y Campeón son utilizadas por los agricultores en la zona de Tumbaco, mientras Centenario es una variedad del INIAP de buena adaptación a la zona. En cuanto a los insumos se utilizó el inoculante biológico con nombre comercial NITO, que posee una concentración de $2,2 * 10^{8} \mathrm{UFC} \mathrm{ml}^{-1}$ de la rizobacteria $B$. subtilis. Previamente a la siembra se incorporó al suelo $5 \mathrm{t} \mathrm{ha}^{-1}$ de compost.

\subsection{Métodos}

En la parcela grande de $54 \mathrm{~m}^{2}$ (3,6 $\mathrm{m}$ de ancho x $15 \mathrm{~m}$ de largo), se aplicaron las dosis de $B$. subtilis, $250 \mathrm{ml}$ y $500 \mathrm{ml}$ en 200 litros de agua ha ${ }^{-1}$, mientras la dosis cero fue el testigo sin aplicación. En las subparcelas de 18 $\mathrm{m}^{2}$ (15 m de largo por 1,2 $\mathrm{m}$ de ancho) se sembraron las variedades. La superficie por repetición fue de $162 \mathrm{~m}^{2}$ para un total de $486 \mathrm{~m}^{2}$ de área total del ensayo. En cada subparcela se sembraron 3 hileras de fréjol separadas a $0,2 \mathrm{~m}$ entre ellas. Se depositó una semilla por sitio cada $0,3 \mathrm{~m}$ para un total de 153 semillas. Se colocaron surcos borde alterador del ensayo.

\subsection{Aplicación de Bacilus subtilis}

Antes de la siembra se aplicó en todo el terreno $500 \mathrm{ml}$ de B. subtilis disueltos en 200 litros de agua con regaderas de mano. Este procedimiento se realizó dos veces, con el fin de nutrir el suelo con los microorganismos. Posteriormente, las respectivas aplicaciones de las dosis de $B$. subtilis se realizaron cada 15 días desde la siembra hasta el momento de floración (R6), con un total de 4 aplicaciones durante el ciclo.

\subsection{Descripción de los tratamientos y variables evaluadas}

La siembra se efectuó el 27 de agosto de 2019. Se evaluaron 9 tratamientos (Tabla 1) y 3 repeticiones en 27 unidades experimentales. Se realizó la siembra depositando una semilla por golpe en tres hileras bajo el sistema de tresbolillo en cada platabanda, para un total de 1.377 semillas empleadas por variedad. Durante el ciclo del cultivo se registraron las variables agronómicas enunciadas en la Tabla 1.

Tabla 1. Tratamientos y variables agronómicas consideradas en el estudio

Table 1. Treatments and agronomic variables included in the study

\begin{tabular}{|c|c|}
\hline Tratamientos & Variables agronómicas \\
\hline $\begin{array}{ll}\text { - } & \text { Tratamiento } 1 \text { (T1): } 0 \mathrm{ml}+\text { Paragachi } \\
\text { - } & \text { Tratamiento } 2 \text { (T2): } 0 \mathrm{ml}+\text { Campeón } \\
\text { - } & \text { Tratamiento } 3 \text { (T3): } 0 \mathrm{ml}+\text { Centenario } \\
\text { - } & \text { Tratamiento } 4 \text { (T4): } 250 \mathrm{ml}+\text { Paragachi } \\
\text { - } & \text { Tratamiento } 5 \text { (T5): } 250 \mathrm{ml}+\text { Campeón } \\
\text { - } & \text { Tratamiento } 6 \text { (T6): } 250 \mathrm{ml}+\text { Centenario } \\
\text { - } & \text { Tratamiento } 7 \text { (T7): } 500 \mathrm{ml}+\text { Paragachi } \\
\text { - } & \text { Tratamiento } 8 \text { (T8): } 500 \mathrm{ml}+\text { Campeón } \\
\text { - } & \text { Tratamiento } 9 \text { (T9): } 500 \mathrm{ml}+\text { Centenario }\end{array}$ & $\begin{array}{l}\text { - Días a la cosecha (DC), comienza con la siem- } \\
\text { bra hasta el momento en que el } 75 \% \text { del en- } \\
\text { sayo presentó granos secos. } \\
\text { - Número de vainas planta-1 (VP) tomando } 10 \\
\text { plantas al azar de la parcela neta. } \\
\text { - Número de granos vaina-1 }(\mathrm{GV}) \text {, de } 40 \text { vainas } \\
\text { tomadas al azar. }\end{array}$ \\
\hline
\end{tabular}

\section{Resultados y discusión}

\subsection{Análisis estadístico}

En la variable DC existió diferencias altamente significativas para el factor variedades. En la comparación de medias para la variable DC se observan tres rangos de significación, donde Centenario posee la media más 
alta con 132 días, siendo la más tardía; y Campeón y Paragachi, con 112 y 120 días, respectivamente, las más precoces (Figura 1). En la variable VP se evidencia diferencias significativas para el factor dosis, igual que para la interacción dosis x variedades (Tabla 2). En relación con los polinomios ortogonales para el factor dosis existió significancia estadística en la tendencia lineal, lo que indica que una mayor dosificación de $B$. subtilis incrementará las VP. En cuanto a los contrastes ortogonales existió diferencias altamente significativas para el contraste de las variedades V2 (Campeón) vs. V3 (Centenario).

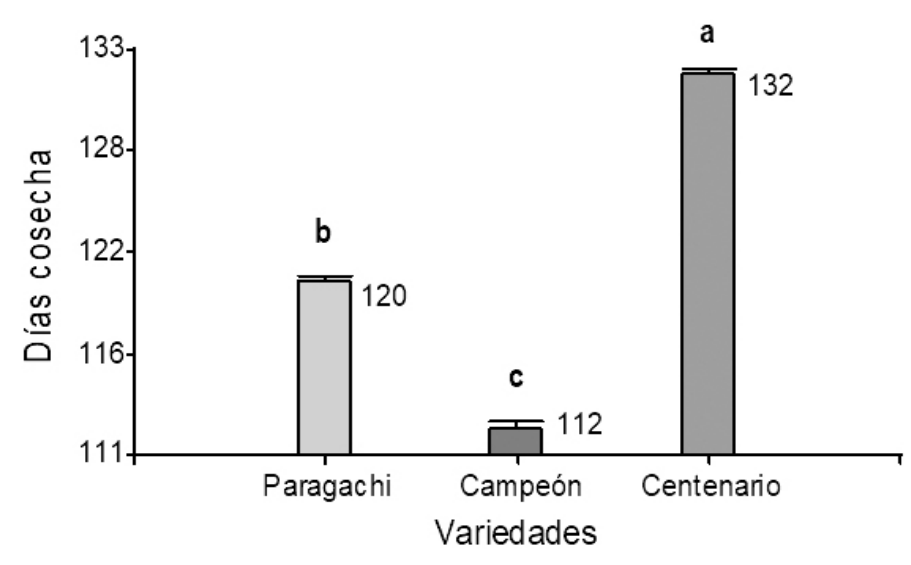

Figura 1. Comparación de medias DC.

Figure 1. Mean comparison of DC.

Tabla 2. Análisis de variación de 6 variables agronómicas registradas en el ensayo de 3 variedades de fréjol con la aplicación de 3 dosis de B. subtilis. Tumbaco 2019.

Table 2. Analysis of variance of 6 agronomic variables recorded in the trial of 3 bean varieties with the application of 3 doses of $B$. subtilis. Tumbaco 2019.

\begin{tabular}{lcccccc}
\hline \multicolumn{1}{c}{ Fuentes de variabilidad } & gl & DC & VP & GV & PGS & RG \\
\hline Bloques & 2 & 1,44 & 17,15 & 0,11 & 15,44 & $12.224,74$ \\
Dosis & 2 & $0,78 \mathrm{~ns}$ & $121,04^{*}$ & $0,11 \mathrm{~ns}$ & $6,78 \mathrm{~ns}$ & $2.509 .206,98^{*}$ \\
Lineal & 1 & $1,39 \mathrm{~ns}$ & $242,00^{*}$ & $0,06 \mathrm{~ns}$ & $14,22 \mathrm{~ns}$ & $4.792 .102,33^{*}$ \\
Cuadrática & 1 & $0,17 \mathrm{~ns}$ & 0,07 & $0,17 \mathrm{~ns}$ & $16,67 \mathrm{~ns}$ & $226.311,62 \mathrm{~ns}$ \\
Error A & 4 & 0,89 & 5,81 & 0,06 & 20,06 & $30.172,05$ \\
Variedades & 2 & $908,33^{* *}$ & 102,37 & $2,11^{*}$ & $464,33^{*}$ & $1.283 .987,05^{* *}$ \\
V1 vs. V2V3 & 1 & $1.204,17^{* *}$ & $4,74 \mathrm{~ns}$ & $0,67 *$ & $416,67 *$ & $36.731,59 \mathrm{~ns}$ \\
V2 vs. V3 & 1 & $612,5^{* *}$ & $200,00^{* *}$ & $3,56^{*}$ & $512,00^{*}$ & $2.531 .242,5^{* *}$ \\
Interacción D x V & 4 & $0,28 \mathrm{~ns}$ & $14,70^{*}$ & $0,06 \mathrm{~ns}$ & $8,11 \mathrm{~ns}$ & $84.542,72^{*}$ \\
Error B & 12 & 1,07 & 3,54 & 0,13 & 20,35 & $14.168,41$ \\
Total & 26 & & & & & 6,14 \\
\hline CV (a) \% & & 0,77 & 9,38 & 6,12 & 9,14 \\
CV (b) \% & & 0,85 & 7,32 & 13,61 & 9,21 & 4,63 \\
\hline
\end{tabular}

$*=$ significativo al $5 \%, * *=$ significativo al $1 \%, \mathrm{~ns}=$ no significativo.

DC: Días a la cosecha, VP: Vainas planta-1 ${ }^{-1}$ GV: Grano vaina ${ }^{-1}$, PGS: Peso grano seco g, RG: Rendimiento grano kg.

En la prueba de Tukey al 5 \% para la interacción se observó cuatro rangos de significación, en donde la interacción de la D3 con Centenario generó un promedio de $34 \mathrm{VP}$, siendo la mejor, seguido por D3 en interacción con la Campeón con un promedio de 29 VP. Esto demuestra que, en este ensayo, la aplicación de B. subtilis incrementa 9 VP en Centenario y 6 VP Campeón en comparación con el testigo. En cambio, Paragachi es superior en $7 \mathrm{VP}$ con la D2 con respecto al testigo D1 (Figura 2). 


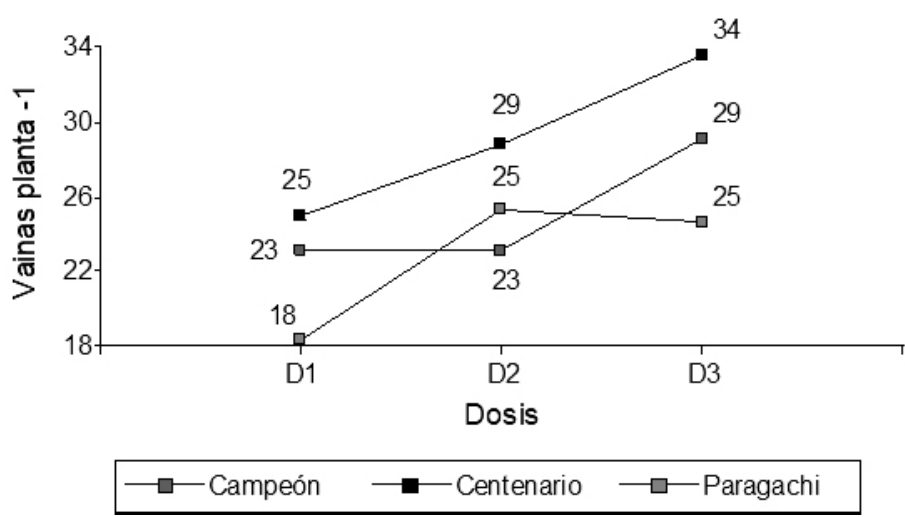

Figura 2. Gráfico particionado de puntos de la interacción en la variable VP.

Figure 2. Partitioned dot plot of the interaction in the VP variable.

Las variables GV y PGS presentaron diferencias significativas para el factor variedades. En la comparación de medias de la variable GV para el factor variedades se encontró dos rangos de significación estadística; en el primer el rango se ubican Centenario y Campeón con un promedio de 5 y $4 \mathrm{GV}$, respectivamente, y en el segundo rango, Paragachi con una media de 3 GV (Figura 3). En la variable PGS se observó dos rangos de significación. En el primer rango, Centenario con 57 g; en el segundo rango, Campeón y Paragachi con 46 y $43 \mathrm{~g}$, respectivamente (Figura 4).

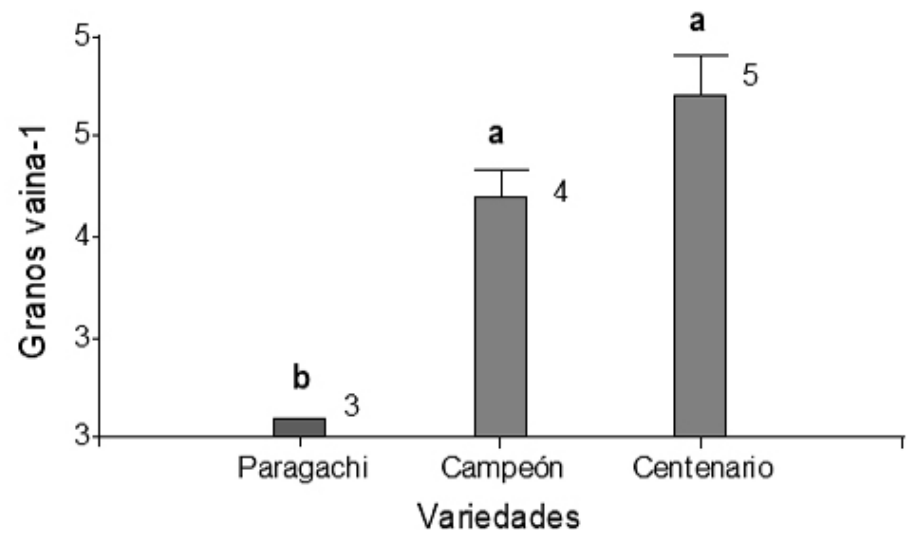

Figura 3. Comparación de medias GV.

Figure 3. Mean comparison of $G V$.

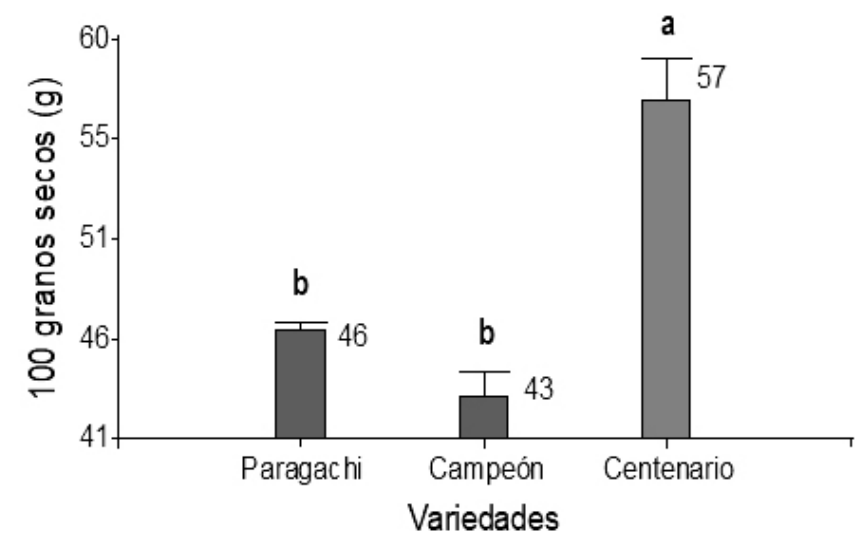

Figura 4. Comparación de medias PGS.

Figure 4. Mean comparison of $P G S$. 
La variable $R G$ presentó diferencia altamente significativa para el factor variedades, mientras que para el factor dosis y la interacción D x V diferencias significativas. Los polinomios ortogonales indicaron que para el factor dosis existe significancia estadística cuando la tendencia es lineal, esto demuestra que un aumento en la dosificación de $B$. subtilis, tendrá como resultado un aumento en el rendimiento de cada variedad. En cuanto a los contrastes ortogonales se observó que existen diferencias estadísticas altamente significativas para el contraste V2 (Campeón) vs. V3 (Centenario) (Tabla 2).

En la prueba de Tukey al $5 \%$ para la interacción en la variable RG, se observa cinco rangos de significación, siendo la interacción D3 x Centenario la de mayor rendimiento con $3.764 \mathrm{~kg} \mathrm{ha}^{-1}$, en tanto que interacción D1 (testigo) x Paragachi es la de menor rendimiento con $2.650 \mathrm{~kg} \mathrm{ha}^{-1}$ (Figura 5).

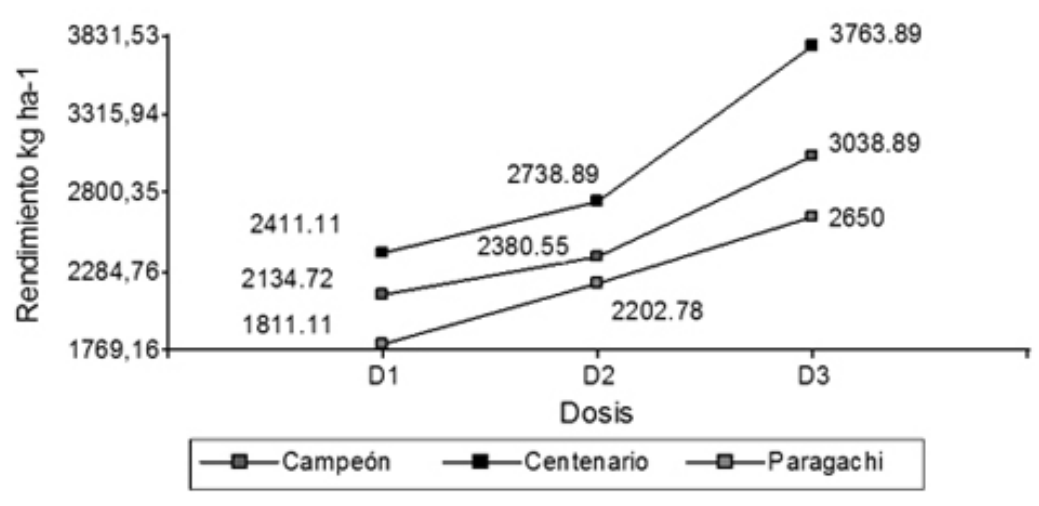

Figura 5. Gráfico particionado de puntos de la interacción en la variable RG.

Figure 5. Partitioned dot plot of the interaction in the GR variable.

\subsection{Análisis no estadístico}

\subsubsection{Análisis químico de suelo}

En la Tabla 3 se observa que el cultivo disminuye la conductividad eléctrica en las tres dosis y el fósforo en la dosis 1 (testigo). Sin embargo, la aplicación de $B$. subtilis aplicado en pre siembra y en las dosis 2 y 3 incrementa el $\mathrm{pH}$, la materia orgánica, el nitrógeno total, el fósforo (respecto al testigo) y el potasio (en todos los tratamientos).

Tabla 3. Características químicas del suelo en pre siembra y pos cosecha.

Table 3. Soil chemical characteristics pre-sowing and post-harvest.

\begin{tabular}{lcccccc}
\hline \multicolumn{1}{c}{ Tratamientos } & $\mathbf{p H}$ & $\mathbf{C E}\left(\mathbf{d s ~ m}^{-1}\right)$ & MOS (\%) & NT (\%) & P (ppm) & K (cmol kg-1) \\
\hline $\begin{array}{l}\text { Pre siembra } \\
\text { Pos cosecha }\end{array}$ & 7,43 & 0,60 & 1,28 & 0,06 & 368,40 & 0,06 \\
- Dosis 2 $(250 \mathrm{ml})$ & $7,65 \pm 0,11$ & $0,34 \pm 0,03$ & $1,72 \pm 0,20$ & $0,08 \pm 0,012$ & $321,70 \pm 13,42$ & $0,55 \pm 0,15$ \\
- Dosis 2 $(250 \mathrm{ml})$ & $7,63 \pm 0,01$ & $0,38 \pm 0,02$ & $2,18 \pm 0,30$ & $0,11 \pm 0,015$ & $351,90 \pm 12,90$ & $0,69 \pm 0,07$ \\
- Dosis 3 $(500 \mathrm{ml})$ & $7,77 \pm 0,03$ & $0,34 \pm 0,03$ & $1,91 \pm 0,30$ & $0,10 \pm 0,015$ & $351,97 \pm 27,67$ & $0,63 \pm 0,20$ \\
\hline
\end{tabular}

El incremento de la materia orgánica y el nitrógeno podría deberse a que el fréjol fija nitrógeno e incorpora sus nódulos al suelo luego de la floración. En cambio, la bacteria solubiliza el fósforo y el potasio presentes en el suelo. La concentración de fósforo disponible en el suelo, según el análisis efectuado antes y después de la cosecha, se observó disminución en las ppm de fósforo, teniendo en cuenta que el cultivo absorbe el elemento para incorporar en diferentes procesos fisiológicos y necesita aproximadamente $9 \mathrm{~kg} \mathrm{ha}^{-1}$ para la formación de 
tallos, flores y vainas (Instituto Interamericano de Cooperación para la Agricultura [IICA], 2009). Haciendo una comparativa del fósforo disponible entre dosis se observa que las D2 y D3 tuvieron mayor concentración en el suelo que en la D1. Este aumento se debió posiblemente al efecto sinérgico de $B$. subtilis con microorganismos nativos del suelo, los cuales potencializaron la disponibilidad de fósforo (Cisneros Rojas et al., 2017).

B. subtilis tiene la capacidad de solubilizar el fósforo por acción de enzimas o ácidos orgánicos (Prashar et al., 2014). La solubilización se da por la producción de enzimas fosfatasas y fitasas generadas por la bacteria, las mismas que alcalinizan el suelo (Fernández y Rodríguez, 2005). Por lo que se podría afirmar que el fósforo solubilizado en esta investigación fue el orgánico. Calero Hurtado et al. (2019), mencionan que desde el punto de vista fisiológico B. subtilis incrementa la capacidad fotosintética de los cultivos, así como su capacidad para absorber agua. También $B$. subtilis interviene en la absorción de nutrientes por medio de la producción de fitohormonas como las auxinas (ácido indol acético), las cuales aumentan la longitud y densidad de las raíces (Barreto Figueiredo et al., 2011).

\subsubsection{Aislamientos de la raíz}

Otro de los factores que limitan los rendimientos del fréjol es la incidencia de enfermedades radiculares causadas por hongos fitopatógenos (Cruz-Triana et al., 2018). Venegas et al. (2005), señalan que Bacillus sp., genera un efecto de antagonismo en el desarrollo de patógenos. Rodríguez Sánchez et al. (2016), indican que B. subtilis usa diferentes procesos dependiendo del hongo fitopatógeno a controlar, entre los que destacan la producción de lipopéptidos, enzimas líticas, sideróforos y activación de la resistencia sistémica inducida. En la etapa de llenado de grano se tomaron muestras de la raíz de cada variedad y se identificaron en el laboratorio de Fitopatología de la Facultad las especies de hongos presentes. Las tres variedades presentaron hongos patógenos y benéficos: Fusarium spp., Rhizoctonia spp., Cylindracarpon sp., Cladosporium sp. y Trichoderma sp (Tabla 4).

Tabla 4. Hongos radiculares patógenos y benéficos de las raíces aisladas en PDA.

Table 4. Pathogenic and beneficial root fungi isolated from roots in PDA.

\begin{tabular}{lcl}
\hline \multicolumn{1}{c}{ Variedad } & Parte aislada & \multicolumn{1}{c}{ Resultado } \\
\hline Paragachi & Raíz & Fusarium spp. Rhizoctonia spp. \\
Campeón & Raíz & $\begin{array}{l}\text { Trichoderma } \text { sp. Rhizoctonia } \text { sp. } \\
\text { Cylindrocarpon } \text { sp. Fusarium } \text { spp. }\end{array}$ \\
Centenario & Raíz & $\begin{array}{l}\text { Fusarium } \text { spp. Cladosporium sp. } \\
\text { Cylindrocarpon } \text { sp. }\end{array}$ \\
\hline
\end{tabular}

En la etapa de llenado de vainas se tuvo alta presencia de saltón de hoja (Empoasca kraemeri) lo que permitió observar el menor daño en Centenario en comparación con Paragachi y Campeón. También hubo presencia de Antracnosis (C. lindemuthianum) en la vaina, observándose sanidad en Centenario y enfermedad en Paragachi y Campeón (Tabla 5).

Tabla 5. Variables adicionales registradas en el ensayo. Tumbaco 2020.

Table 5. Additional variables recorded in the trial. Tumbaco 2020.

\begin{tabular}{lccccccc}
\hline Variedad & Floración & $\begin{array}{c}\text { Color } \\
\text { flor }\end{array}$ & $\begin{array}{c}\text { Vigor* } \\
\mathbf{1 - 9}\end{array}$ & Hábito** & $\begin{array}{c}\text { Antracnosis } \\
\text { vaina }\end{array}$ & $\begin{array}{c}\text { Saltón de } \\
\text { hoja }\end{array}$ & $\begin{array}{c}\text { Madurez } \\
\text { fisiológica }\end{array}$ \\
\hline Centenario & 55 & Lila & 1 & 1 & Resistente & Tolerante & 105 \\
Paragachi local & 53 & Blanco & 7 & $1-2$ & Susceptible & Susceptible & 100 \\
Campeón & 50 & Blanco & 4 & 1 & Susceptible & Intermedio & 95 \\
\hline
\end{tabular}

*1 lo mejor; $* * 1$ determinado, 2 indeterminado 


\subsection{Relación Beneficio/costo}

Tomando como referencia un precio de venta de 1,55, 1,33 y 1,22 USD kg-1 de las variedades de fréjol Paragachi, Campeón y Centenario, respectivamente. Los resultados indican que el mejor tratamiento es el T9 (500 $\mathrm{ml}$ x Centenario) de USD 0,32 por dólar invertido (Figura 6).

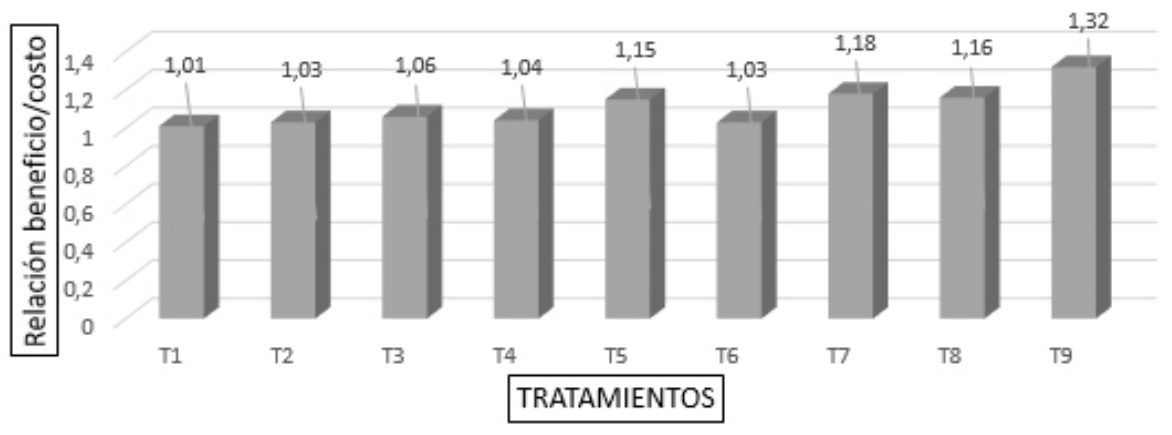

Figura 6. Beneficio/costo de tratamientos.

Figure 6. Benefit/cost of treatments.

\section{Conclusiones}

Se evidenció un aumento del rendimiento con el uso de la D3 de B. subtilis en comparación con el testigo para todas las variedades estudiadas. El rendimiento se incrementó por acción de las rizobacterias al solubilizar fósforo. Se asume que las rizobacterias protegieron las raíces del ataque de hongos fitopatógenos del suelo.

B. subtilis juega un papel importante en la absorción de fósforo, pues la concentración de fósforo al final del estudio fue mayor para la D3, con $352 \mathrm{ppm}$. De esta forma, el nutriente se encontrará disponible en el suelo y en mayor concentración en comparación con la parcela testigo para el siguiente cultivo. La variedad que tuvo un mejor rendimiento con el uso de la D3 de B. subtilis fue Centenario con un rendimiento de 3.764 $\mathrm{kg} \mathrm{ha}^{-1}$, seguida de Campeón con 3.038,89 $\mathrm{kg} \mathrm{ha}^{-1}$, y finalmente Paragachi con $2.650 \mathrm{~kg} \mathrm{ha}^{-1}$. El uso de las tres variedades y la aplicación de la $\mathrm{D} 3$ de $B$. subtilis generó también una mayor rentabilidad.

\section{Agradecimientos}

Los autores agradecen a Dundas Corporation Powerdundas S.A. por el apoyo en el desarrollo de la presente investigación mediante la donación y capacitación en el uso de NITO que contiene Bacillus subtilis.

\section{Contribuciones de los autores}

- Melanie Arline Chávez Rea: conceptualización, investigación, metodología, recursos, redacción - borrador original.

- José Eliecer Vásquez-Guzmán: validación, redacción - revisión y edición.

\section{Referencias}

Akibode, S., \& Maredia, M. (2011). Global and regional trends in production, trade and consumption of food legume crops. Report submitted to SPIA. Department of agricultural, food and resources economics. Michigan State University

Armenta-Bojórquez, A. D., García-Gutiérrez, C., Camacho-Báez, J. R., Apodaca-Sánchez, M. A., Gerardo-Montoya, G., \& Nava-Pérez, E. (2010). Biofertilizantes en el desarrollo agrícola en México. Ra Ximhai, 6(1), 51-56. http://revistas.unam.mx/index.php/rxm/article/view/17885 
Barreto Figueiredo, M. V., Seldin, L., de Araujo, F. F., \& Ramos Mariano, R. L. (2010). Plant Growth Promoting Rhizobacteria: Fundamentals and Applications. In D. K. Maheshwari (ed.) Plant Growth and Health Promoting Bacteria, Microbiology Monographs, vol 18 (21-43). Springer. https://doi.org/10.1007/9783-642-13612-2_2

Calero Hurtado, A., Quintero Rodríguez, E., Pérez Díaz, Y., Olivera Viciedo, D., Peña Calzada, K., \& Jiménez Hernández, J. (2019). Efecto entre microorganismos eficientes y fitomas-e en el incremento agroproductivo del frijol. Biotecnología en el Sector Agropecuario y Agroindustrial, 17(1), 25-33. https://doi. org/10.18684/bsaa.v17n1.1201

Cisneros Rojas, C. A., Sánchez de Prager, M., \& Menjivar Flores, J. C. (2017). Identificación de bacterias solubilizadoras de fosfatos en un Andisol de la región cafetera colombiana. Revista Colombiana de Biotecnología, 19(1), 21-28. https://doi.org/10.15446/rev.colomb.biote.v19n1.65966

Corrales Ramírez, L., Caycedo Lozano, L., Gómez Méndez, M., Ramos Rojas, S., \& Rodríguez Torres, J. (2017). Bacillus spp: una alternativa para la promoción vegetal por dos caminos enzimáticos. NOVA, 15(27), 45-65. https://doi.org/10.22490/24629448.1958

Cruz-Triana, A., Rivero-González, D., Infante-Martínez, D., Echevarría-Hernández, A., Martínez-Coca, B. (2018). Manejo de hongos fitopatógenos en Phaseolus vulgaris L. con la aplicación de Trichoderma asperellum Samuels, Lieckfeldt y Nirenberg. Revista de Protección Vegetal, 33(3), 1-7. http://revistas. censa.edu.cu/index.php/RPV/article/view/975

Fernández, M. T., \& Rodríguez, H. (2005). El papel de la solubilización de fósforo en los biofertilizantes microbianos. ICIDCA. Sobre los Derivados de la Caña de Azúcar, XXXIX(3), 27-34.

Garcés Fiallos, F. R., Aguirre Calderón, Á. J., Díaz-Ocampo, E., Sánchez Mora, F. D., Prieto Benavides, O., \& Garcés-Estrella, R. (2014). Enfermedades y componentes de rendimiento en dieciséis genotipos de fréjol en Quevedo, Ecuador. Ciencia y Tecnología, 6(2), 31-39. https://doi.org/10.18779/cyt.v6i2.130

Garcés Fiallos, F. R., Zabala Palacios, R. G., Díaz Coronel, T. F., \& Vera Avilés, D. F. (2012). Evaluación agronómica y fitosanitaria de germoplasma de fréjol (Phaseolus vulgaris L.) en el trópico húmedo ecuatoriano. Revista UDO Agrícola, 12(2), 230-240.

Instituto Interamericano de Cooperación para la Agricultura [IICA]. 2009. Guía técnica para el cultivo de frijol en los municipios de Santa Lucía, Teustepe y San Lorenzo del Departamento de Boaco, Nicaragua. IICA-RED SICTA-COSUDE

Instituto Nacional de Estadística y Censos [INEC], 2019. Estadísticas agropecuarias. ESPAC. https://www. ecuadorencifras.gob.ec/estadisticas-agropecuarias-2/

Llanos, M. (2017). Bacterias solubilizadoras de fosfato del género Bacillus en suelos de la provincia de El Collao (Puno) y su efecto en la germinación y crecimiento de quinua (Chenopodium quinoa Willd.) en condiciones de invernadero. Universidad Nacional del Altiplano.

Prashar, P., Kapoor, N., \& Sachdeva, S. (2014). Rhizosphere: its structure, bacterial diversity and significance. Reviews in Environmental Science and Bio/Technology, 13, 63-77. https://doi.org/10.1007/s11157-0139317-z

Rodríguez Sánchez, J., Ríos Rocafull, Y., \& Baró Robaina, Y. (2016). Efectividad de cepas de Azotobacter sp. y Bacillus sp. para el control de especies fúngicas asociadas a hortalizas. Cultivos Tropicales, 37(Supl. 1), 13-19.

Terry Alfonso, E., Leyva, Á., \& Hernández, A. (2005). Microorganismos benéficos como biofertilizantes eficientes para el cultivo del tomate (Lycopersicon esculentum, Mill). Revista Colombiana de Biotecnología, 7(2), 47-54. https://revistas.unal.edu.co/index.php/biotecnologia/article/view/498

Torres Navarrete, E. D., Sánchez Laiño, A., Cedeño Briones, A., Haro Chong, A., Torres Navarrete, A., González Osorio, B., Quisphe Caiza, D., \& Reyes Bermeo, M. (2014). Caracterización de la producción de frijol en la provincia de Cotopaxi-Ecuador: Caso comuna Panyatug. Ciencia y Tecnología, 6(1), 23-31. https://doi.org/10.18779/cyt.v6i1.126

Venegas, G., E., Ciampi, P., L., Collado, G., L., Costa, L., M., Fuentes, P., R., Nissen, M., J., Schobitz, T., R., \& Schoebitz, C., M. (2005). Aislamiento e identificación de bacterias nativas del género Bacillus Cohn, antagonistas de cepas patógenas de Fusarium link en cala. Agro Sur, 33(2), 1-12. https://doi.org/10.4206/ agrosur.2005.v33n2-01

Vidal, J. (2003). Dinámica del potasio en el suelo y su requerimiento por los cultivos. Colegio de postgraduados, Institución de enseñanza e investigación en ciencias agrícolas. 